\title{
Technological challenges for CW operation of small-radius semiconductor ring lasers
}

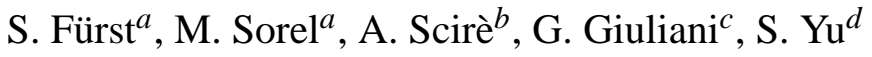 \\ ${ }^{a}$ Department of Electronics and Electrical Engineering, \\ University of Glasgow, G12 8LT, Glasgow, United Kingdom; \\ ${ }^{b}$ Institut Mediterrani d'Estudis Avançats, Illes Balears, Spain; \\ ${ }^{c}$ Università degli Studi di Pavia, 1 Via Ferrata, Pavia, Italy; \\ ${ }^{d}$ University of Bristol, Merchant Venturers Building, \\ Woodland Road, Bristol, United Kingdom
}

\begin{abstract}
Theoretical investigation and device measurements are reported to demonstrate the strict fabrication requirements of small diameter shallow etched semiconductor ring lasers. A very accurate control over the dry etching depth is crucial to both minimise the bending losses and achieve very precise control of the coupling ratio in directional couplers. A reactive ion etching process was developed on Aluminium-quaternary wafer structures, showing selectivity greater than 30 between the AlInAs core layer and the InP upper cladding. The process proved very effective in providing a complete and controllable etching of directional couplers with $500 \mathrm{~nm}$ wide gaps. Assessment on the effect of the bending losses and on the minimum ring radius was performed through characterisation of half ring lasers. A minimum current threshold of $34 \mathrm{~mA}$ is reported on $150 \mu \mathrm{m}$ ring radius devices emitting at $1300 \mathrm{~nm}$.
\end{abstract}

Keywords: semiconductor ring laser, bending loss, selective dry etching, aluminium quaternary

\section{INTRODUCTION}

Semiconductor ring lasers (SRLs) have been receiving growing interest since the early 1990s because of their potential for monolithical integration. Recently, accurate investigations on the bistable behaviour between the two counterpropagating modes $^{1,2}$ have paved the way for their development as optical flip-flops in all-optical communication systems and optical digital processing. ${ }^{3}$ Reducing the ring radius of the SRLs is not only appealing for the footprint of the devices but becomes a key issue in the fabrication of very fast optical bistables since the switching speed between the counterpropagating modes is related to the cavity dimension. In this down scaling process, however, the bending losses pose a severe limitation to the minimum dimension of the devices. In very small radius SRLs (a few tens of microns), a deep etched waveguide approach is necessary to increase the mode confinement. This approach, compared to a more conventional shallow etched waveguide configuration, is very challenging in terms of sidewall scattering loss, output power coupling mechanism, device degradation and waveguide fabrication tolerance to ensure single-transverse mode operation.

In this paper, we investigate the miniaturisation of SRLs in shallow etched waveguide devices. From numerical simulations, it appears that bending losses are extremely sensitive to the waveguide etching depth. In order to guarantee a very precise control over the etching depth, a reactive ion etching (RIE) process was developed with a high selectivity between the upper cladding (InP) and the core layer (AlInAs). The process provides very smooth surfaces and allows a complete etch of the gap between the waveguides in a directional coupler, which enables a precise control in the output power coupling.

Further author information: (Send correspondence to S.F.)

S.F.: E-mail: s.furst@elec.gla.ac.uk, Telephone: +44 (0)141 3308631

M.S.: E-mail: m.sorel@elec.gla.ac.uk, Telephone: +44 (0)141 3305229

A.S.: E-mail: scire@imedea.uib.es, Telephone: +34 (0)971 171314

G.G.: E-mail: guido.giuliani@unipv.it, Telephone: +39 0382985224

S.Y.: E-mail: s.yu@ bristol.ac.uk, Telephone: +44 (0)117 9545295

Semiconductor Lasers and Laser Dynamics II, edited by Daan Lenstra, Markus Pessa, lan H. White, Proc. of SPIE Vol. 6184, 61840R, (2006) · 0277-786X/06/\$15 · doi: 10.1117/12.667588 


\section{THEORY AND SIMULATION}

The wafer structure used to fabricate the devices is a multiple quantum well (QW) Aluminium quaternary material system $\left(\mathrm{Al}_{x} \mathrm{Ga}_{y} \mathrm{In}_{1-x-y} \mathrm{As}-\mathrm{InP}\right)$ emitting at $1.3 \mu \mathrm{m}$. The main advantage of this material over the standard phosphorous quaternary $\left(\mathrm{Ga}_{x} \mathrm{In}_{1-x} \mathrm{As}_{y} \mathrm{P}_{1-y}-\mathrm{InP}\right)$ is the better thermal behaviour because of the reduced carrier leakage, ${ }^{4}$ resulting from a larger conduction band offset ( $\Delta E_{c}=0.72 \Delta E_{g}$ in Al-quaternary; $\Delta E_{c}=0.4 \Delta E_{g}$ in phosphorous quaternary). The QWs are sandwiched between two $50 \mathrm{~nm}$-thick InAlGaAs-InAlAs layers, with the inclusion of two additional $50 \mathrm{~nm}$-thick InAlAs layers to decrease the leakage of electrons from the QWs into the InP cladding layers. ${ }^{5}$ The wafer structure is shown in Table 1.

To gather information on the effective refractive index contrast $\Delta n_{e f f}$, the effective refractive index of the slab mode was calculated using a mode solver with different upper cladding thicknesses. The result is shown in Fig. 1 (plain curve), where $d=0$ corresponds to a situation in which the InP upper cladding outside the waveguide was entirely etched. The effective refractive index difference rapidly increases to a final value of $\Delta n_{e f f}=0.055$ as the upper cladding is completely etched. Another set of simulations was performed to assess the fabrication tolerances of directional couplers on the dry etching depth, employing a $2 D$ beam propagation method (BPM) and the previous results for $\Delta n_{e f f}$. The couplers were designed to provide a theoretical coupling factor value of $70 \%$ when the upper cladding is completely etched away, with $2 \mu \mathrm{m}$ wide waveguides at distances of $1 \mu \mathrm{m}$ and $0.5 \mu \mathrm{m}$ over a length of $390 \mu \mathrm{m}$ and $128 \mu \mathrm{m}$, respectively.

As the etching depth is decreased, the decrease in $\Delta n_{e f f}$ quickly reduces the coupling length and thus increase the coupling factor. Since in the directional coupler there is a periodical exchange of power from one waveguide to the other, for upper cladding thicknesses greater than $65 \mathrm{~nm}$ and a gap of $1 \mu \mathrm{m}$, the outcoupled light starts to couple back into the

Table 1. Al-quaternary material structure used for the fabrication of ring lasers.

\begin{tabular}{|r|l|l|}
\hline Thickness & Composition & Layer \\
\hline $200 \mathrm{~nm}$ & $\mathrm{p}^{+}-\mathrm{In}_{0.53} \mathrm{Ga}_{0.47} \mathrm{As}$ & Cap layer \\
\hline $1650 \mathrm{~nm}$ & $\mathrm{p}-\mathrm{InP}$ & Upper cladding \\
\hline $50 \mathrm{~nm}$ & $\mathrm{In}_{0.52} \mathrm{Al}_{0.48} \mathrm{As}$ & Electron confinement \\
\hline $50 \mathrm{~nm}$ & $\mathrm{In}_{0.52} \mathrm{Al}_{0.4} \mathrm{Ga}_{0.08} \mathrm{As}$ & SCH \\
\hline $6 \times 6 \mathrm{~nm}$ & $\mathrm{In}_{0.73} \mathrm{Al}_{0.165} \mathrm{Ga}_{0.105} \mathrm{As}$ & Strained quantum wells \\
\hline $7 \times 10 \mathrm{~nm}$ & $\mathrm{In}_{0.52} \mathrm{Al}_{0.36} \mathrm{Ga}_{0.12} \mathrm{As}$ & Barriers \\
\hline $50 \mathrm{~nm}$ & $\mathrm{In}_{0.52} \mathrm{Al}_{0.4} \mathrm{Ga}_{0.08} \mathrm{As}$ & SCH \\
\hline $50 \mathrm{~nm}$ & $\mathrm{In}_{0.52} \mathrm{Al}_{0.48} \mathrm{As}$ & Electron confinement \\
\hline$\sim 350 \mu \mathrm{m}$ & $\mathrm{n}-\mathrm{InP}$ & Substrate \\
\hline
\end{tabular}
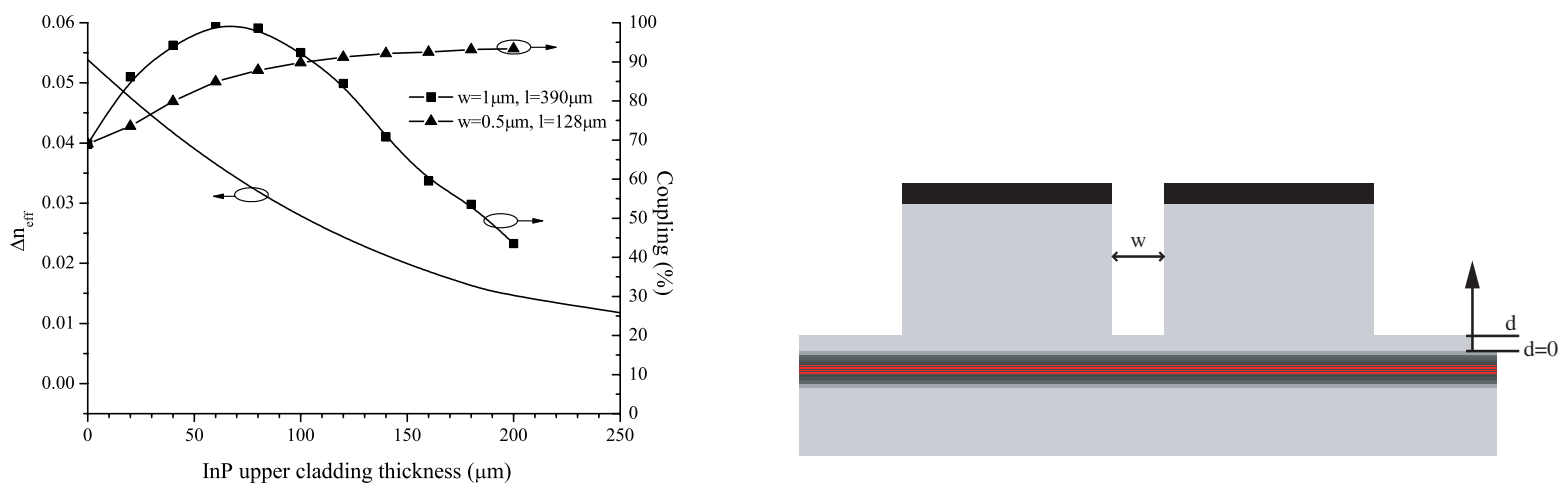

Figure 1. Simulated effective refractive index difference (plain curve) as a function of upper cladding thickness outside the waveguides $(d)$, and output coupling ratio on a directional coupler for two different gaps ( $w=1 \mu \mathrm{m}$ and $0.5 \mu \mathrm{m})$. The initial coupling was set to $70 \%$ corresponding to a coupler length $(l)$ of $390 \mu \mathrm{m}$ and $128 \mu \mathrm{m}$, respectively. $d=0$ corresponds to the top complete etching of the upper cladding. 


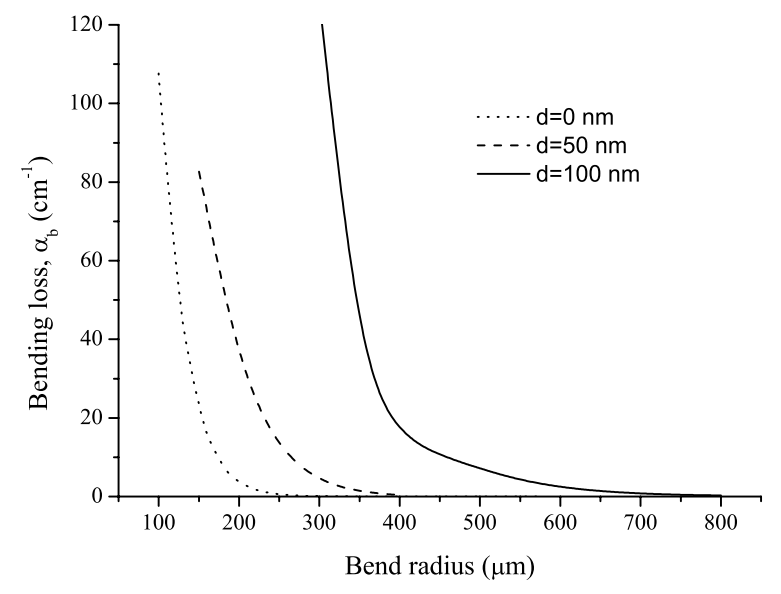

Figure 2. Simulated bending loss as a function of bending radius in $2 \mu \mathrm{m}$-wide waveguides for different etching depth.

input waveguide, decreasing therefore the coupling factor.* An error of only $65 \mathrm{~nm}$ in the depth leads to a change of the coupling ratio from $70 \%$ to $100 \%$, preventing laser action. As the coupler's gap is decreased, the coupling ratio becomes less sensitive to etch depth variations, as shown in Fig. 1 for a gap of $0.5 \mu \mathrm{m}$. Depending on lithographic and etching accuracy, a figure of merit can be defined to provide an optimum gap width for maximum robustness. In our simulations, we chose a gap width of $0.5 \mu \mathrm{m}$ which can be defined by UV lithography with good reproducibility.

A mode calculation was performed on waveguides with different bend radii using a commercial software which combines BPM analysis and a coordinate transformation method to map a curved waveguide onto a straight waveguide. The simulations generate complex effective refractive indexes, from which the bending loss can be calculated (Figure 2). A complete etching of the upper cladding allows negligible bending losses down to a radius of $250 \mu \mathrm{m}$. A decrease in the etching depth of $50 \mathrm{~nm}$ and $100 \mathrm{~nm}$, increases this figure to $400 \mu \mathrm{m}$ and $700 \mu \mathrm{m}$, respectively.

Predicting the performance of the ring lasers (such as threshold current and external quantum efficiency) can be done as follows: the gain of a Fabry-Perot quantum well laser at threshold can be expressed as ${ }^{6}$ :

$$
n \Gamma_{w} g_{t h}=\alpha_{0}+\frac{1}{L} \ln \frac{1}{R},
$$

where $n$ is the number of quantum wells, $\Gamma_{w}$ is the confinement factor per well, $g_{t h}$ is the gain at threshold, $\alpha_{0}$ is the material loss, $L$ is the cavity length and $R$ is the reflectivity of the mirrors. Eqn. (1) is modified for ring lasers to include both curved and straight sections for the couplers (see Figure 3):

$$
(l+2 r \pi) n \Gamma_{w} g_{t h}=L \alpha_{0}+\ln \frac{1}{\cos ^{2} \frac{\pi l}{2 l_{100}}}+2 \pi r \alpha_{b}(r),
$$

where $l$ is the length of the coupler, $l_{100}$ corresponds to half beat length of the directional coupler ( $100 \%$ coupling factor), $r$ is the radius of the curved sections, $L=2 l+2 r \pi$ is the total length of the cavity and $\alpha_{b}(r)$ is the bending loss. The logarithmic term in Eqn. (2) accounts for the loss originated by the output power coupling $\left(\alpha_{c}\right)$ and describes the periodic behaviour of directional couplers as a function of their lengths. In Eqn. (2), it is assumed that the directional coupler is bandgap-shifted and left unpumped to avoid refractive index modulation of the coupler and coupling dependence to the pumping current.

\footnotetext{
${ }^{*}$ It is worth noting that the coupling factor is weakly affected by variations in the outer etching depth but strongly changes as the etching depth in the gap between the waveguides varies.
} 


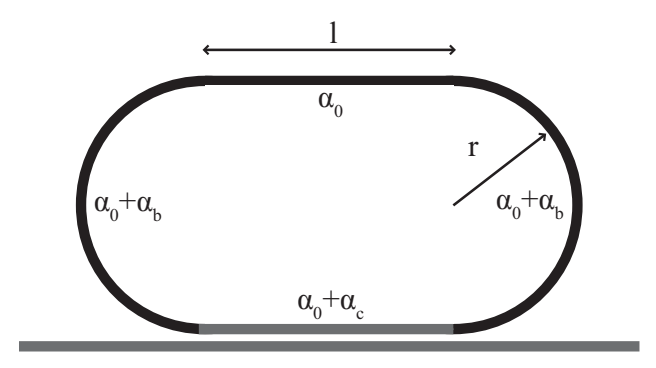

Figure 3. A typical configuration of a racetrack cavity semiconductor ring laser with the different loss factors. The grey part indicates the passive coupler and output waveguides.
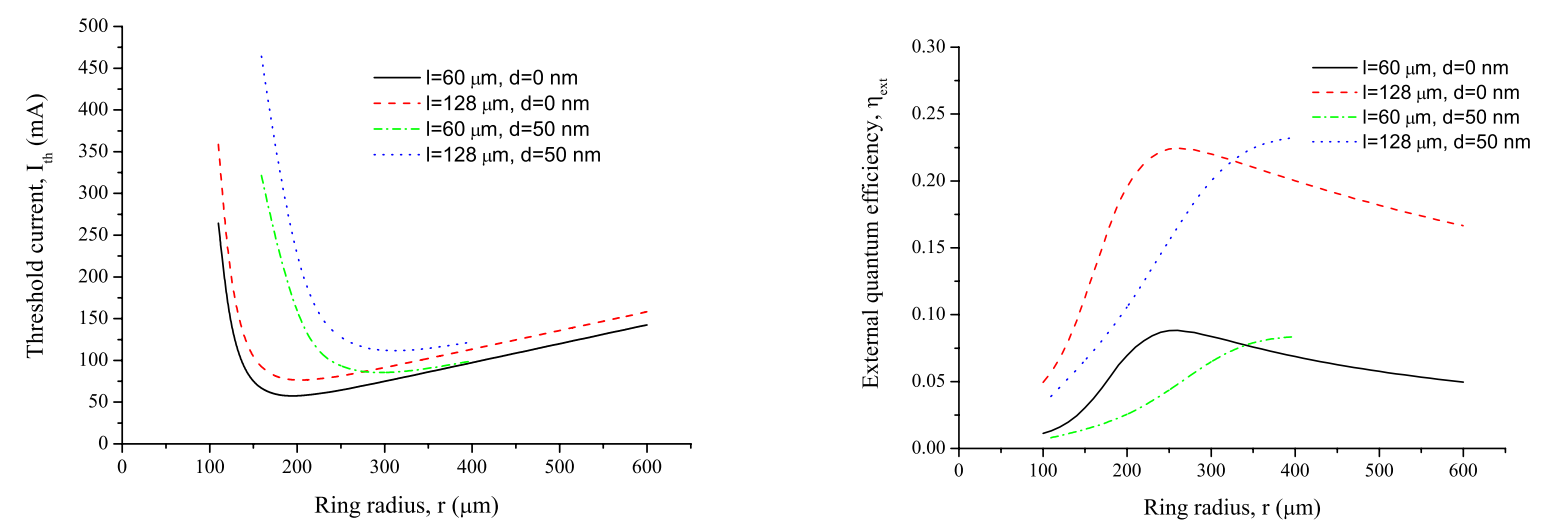

Figure 4. Calculated ring laser performance vs. ring radius for different coupler lengths $(l)$, and for upper cladding thicknesses $(d)$ of 0 and $50 \mathrm{~nm}$. In the case of complete removal of the upper cladding $(d=0)$, the coupling ratio is $20 \%$ and $70 \%$ at coupler lengths $(l)$ of 60 and $128 \mu \mathrm{m}$, respectively.

The threshold current density $\left(J_{t h}\right)$ in multiple quantum well lasers can be approximately related to the threshold gain in the laser cavity. ${ }^{6}$ The external quantum efficiency $\left(\eta_{e x t}\right)$ above threshold is related to the losses and internal quantum efficiency ${ }^{7}$ as follow:

$$
\begin{gathered}
J_{t h}=\frac{J_{t} n}{\eta_{i}} \exp \frac{g_{t h}}{g_{0}} \text { and } \\
\eta_{e x t}=\eta_{i} \frac{\alpha_{c}}{\alpha_{t}} .
\end{gathered}
$$

The internal quantum efficiency $\left(\eta_{i}\right)$, the transparency gain $\left(g_{0}\right)$ and the transparency current density $\left(J_{t} n\right)$ are material parameters, while the threshold gain, the loss introduced by the coupler $\left(\alpha_{c}\right)$ and the total loss $\left(\alpha_{t}\right)$ can be deduced from: Eqn. (2), the simulation results presented in Fig. 2, the ring laser geometry and the material loss. The material parameters can be deduced from well-known material characterization techniques such as broad area and ridge waveguide F-P laser measurements, while the confinement factor $\left(\Gamma_{w} n\right)$ is usually known from simulation.

Threshold currents and external quantum efficiencies were calculated for different ring laser radii and the result is plotted on Fig. 4. ${ }^{\dagger}$ A minimum value in threshold current of $57 \mathrm{~mA}$ is achieved at a radius of approximately $190 \mu \mathrm{m}$ at a coupling factor of $20 \%(w=0.5 \mu \mathrm{m})$. The radius can be further decreased to approximately $150 \mu \mathrm{m}$ without suffering a major penalty in the threshold current $(74 \mathrm{~mA})$. All these figures were calculated with a directional coupler gap of $0.5 \mu \mathrm{m}$. Note that the minimum threshold current is not at the same radius as the maximum external quantum efficiency, so there is a trade-off between the minimum threshold current or the highest quantum efficiency.

\footnotetext{
${ }^{\dagger}$ Assuming $2.5 \mu \mathrm{m}$ wide waveguides, $0.5 \mu \mathrm{m}$ gap for the coupler and the following parameters specific to our material: $n=6$, $g_{0}=359 \mathrm{~cm}^{-1}, J_{t}=87.6 \mathrm{~A} \mathrm{~cm}^{-2}, \eta_{i}=0.408, \alpha_{0}=4 \mathrm{~cm}^{-1}, \Gamma_{w} n=0.1$.
} 


\section{DRY ETCHING OPTIMISATION}

Dry etching optimisation was performed on a pattern comprising several $2.5 \mu \mathrm{m}$ wide optical waveguides and several gratings with periods ranging from $150 \mathrm{~nm}$ to $250 \mathrm{~nm}$. The pattern was written on a PMMA resist by electron-beam lithography and subsequently transferred to a $200 \mathrm{~nm}$ thick $\mathrm{SiO}_{2}$ layer to form a solid dry etching mask.

Control of the etching depth is achieved by using the high selectivity of InP dry etching over InAlAs. ${ }^{8}$ Different etching runs were performed in $\mathrm{CH}_{4} / \mathrm{H}_{2} / \mathrm{O}_{2}$ plasma, varying both the relative flow rate of the gas mixture and the total RF power. Addition of $\mathrm{O}_{2}$ to the gas mixture decreases the etch rate but improves the sidewall verticality because it partially removes the polymer which forms during InP etching. ${ }^{9}$

A preliminary result $\left(7 / 56 / 0.4 \mathrm{sccm}\right.$ flow rate of $\mathrm{CH}_{4} / \mathrm{H}_{2} / \mathrm{O}_{2}$, respectively) showed an $\mathrm{InP}$ etch rate of $55 \mathrm{~nm} / \mathrm{min}$ and a selectivity greater than 10 , but with poor sidewall verticality and a severe undercut in the top layer. In addition, the excess polymer formation gave nonlinear etching rate and therefore unpredictable etch depth.

Finally, a total RF power of $50 \mathrm{~W}$, a pressure of $30 \mathrm{mTorr}$ and a $6 / 54 / 0.6 \mathrm{sccm}$ flow rate $\left(\mathrm{CH}_{4} / \mathrm{H}_{2} / \mathrm{O}_{2}\right)$ gave the best etching results in terms of sidewall verticality. Furthermore, the etch rate on $\mathrm{InP}$ decreased to $35 \mathrm{~nm} / \mathrm{min}$, while the rate on InAlAs was only about $1 \mathrm{~nm} / \mathrm{min}$, corresponding to a selectivity greater than 30 . Very little polymer formation was found, which resulted in a linear etching speed over time as shown in Fig. 5. This means that the InAlAs layer acts as a good dry etching stop-layer enabling a very precise control over the waveguides' etching depth.

The set of parameters used gives very smooth etched surfaces as the etching stops on the InAlAs layer and flattens out any unevenness as shown in Fig. 6. The high selectivity can be explained by the oxidation of the InAlAs layer during the etching, forming a thin layer of $\mathrm{Al}_{2} \mathrm{O}_{3}$. This layer could provide electrical passivation of the dry etched surfaces and improve the device performance.

A major limitation in reactive ion etching processes is that not all of the ions can get into small gaps (such as a coupler), thus resulting in a smaller etching speed, as shown in Fig. 7 (a). The results from our etching tests show that the etching rate inside a gap of $1 \mu \mathrm{m}$ is $10 \%$ slower than the rate on an open surface, and becomes $30 \%$ slower when the gap is reduced to $500 \mathrm{~nm}$. Figure 7 (b) shows SEM pictures of over-etched directional couplers, and the etching profile of a $500 \mathrm{~nm}-$ coupler. A 12-minute over-etching guarantees a complete etching inside the $500-\mathrm{nm}$ wide gap, preserving both the waveguide width and depth.

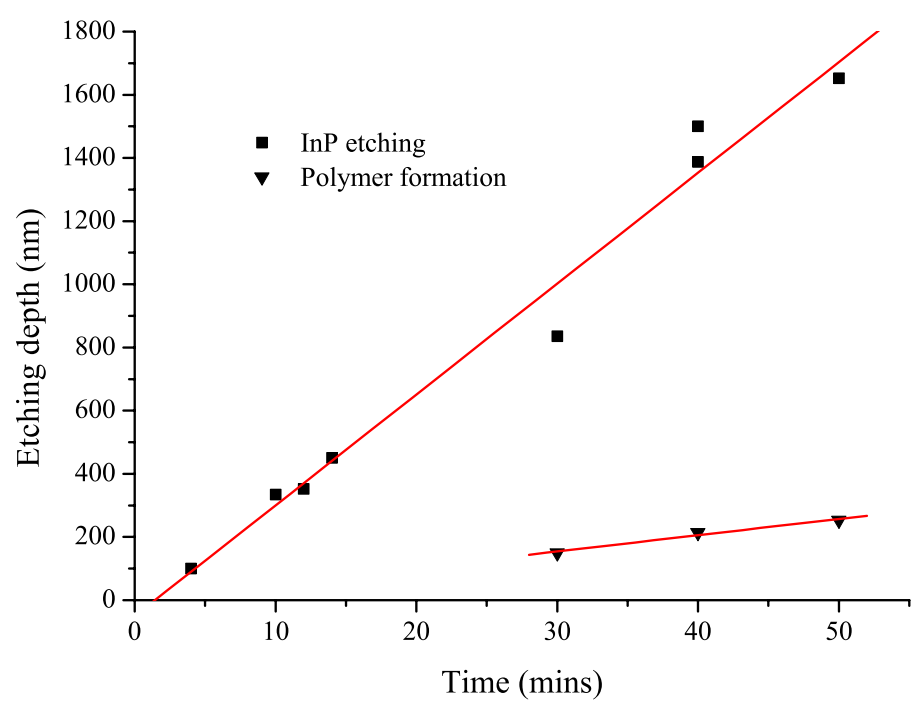

Figure 5. Optimised dry etching characteristics. The fitted curves show a linear etching speed of $35 \mathrm{~nm} / \mathrm{min}$, an induction time of $0.7 \mathrm{~min}$ and a polymer formation rate on the top of the silica mask of $5 \mathrm{~nm} / \mathrm{min}$. 


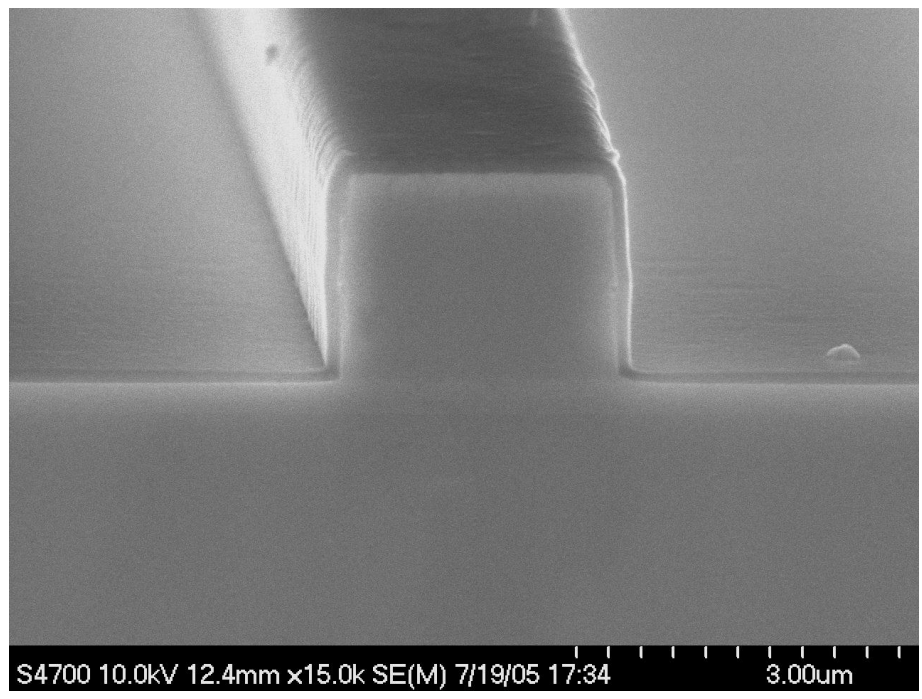

Figure 6. A cross sectional view of a passive waveguide etched down to the InAlAs layer and subsequently covered with $200 \mathrm{~nm}$ PECVD $\mathrm{SiO}_{2}$ (after removing the etching mask) for electrical passivation.

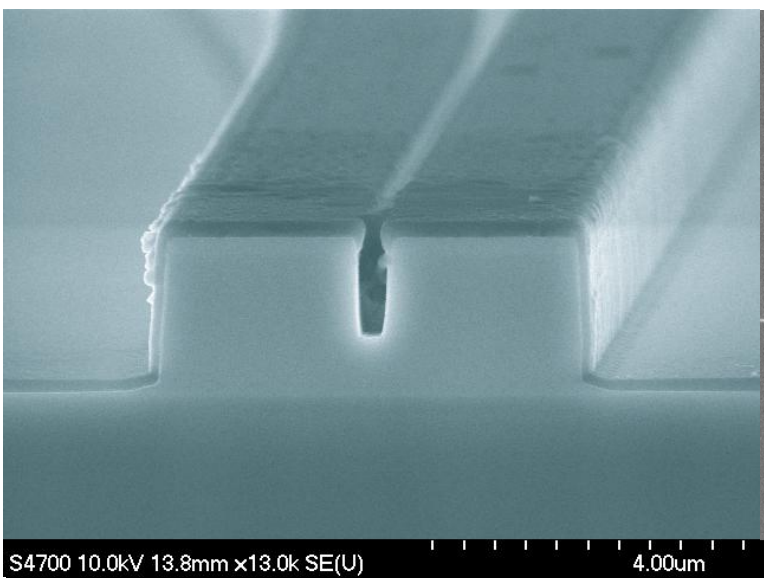

(a) The effect of small gaps on the etching: the coupler is not etched down to the InAlAs layer.

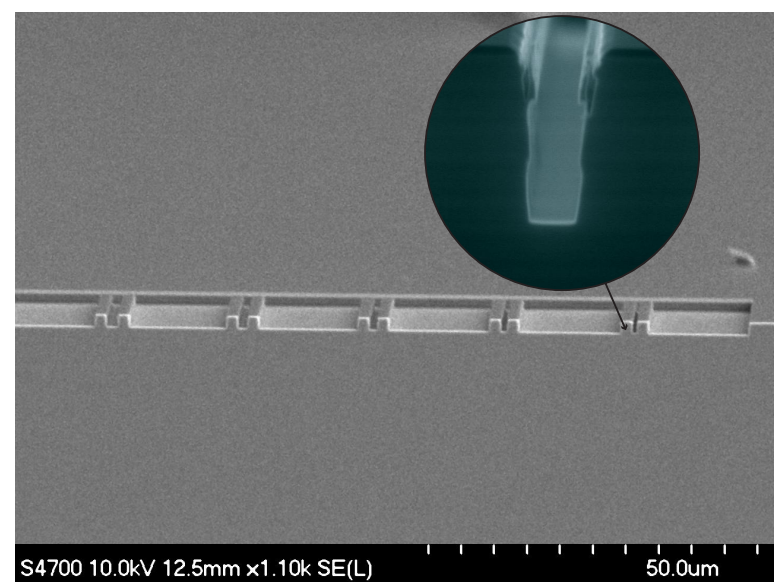

(b) After 12 minutes over-etching, the bottom surface of the coupler flattens as the etching reaches the stop-etch layer.

Figure 7. SEM pictures of dry etched test couplers. 


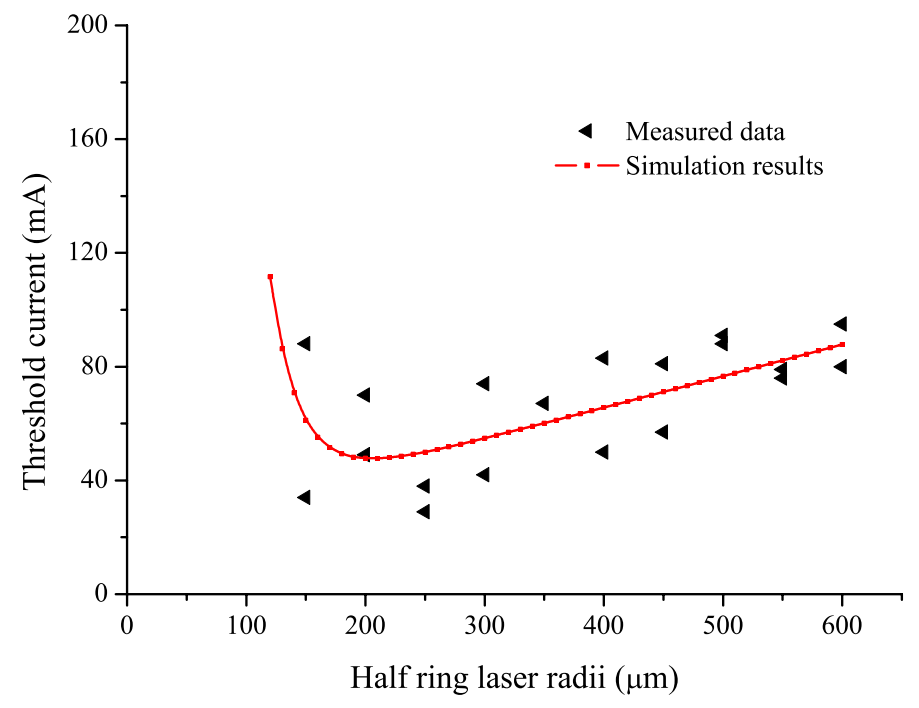

Figure 8. Measured and simulated half ring laser thresholds.

\section{RESULTS}

Several half ring lasers ${ }^{\ddagger}$ were fabricated with ring radii ranging from 150 to $600 \mu \mathrm{m}$ to get information on the bending losses (the length of the straight section was fixed to $l=100 \mu \mathrm{m}$ ). The measured threshold currents are reported in Fig. 8 . In order to model the behaviour of half ring lasers, Eqn. (2) has to be modified as follow:

$$
\operatorname{Ln} \Gamma_{w} g_{t h}=L \alpha_{0}+\ln \frac{1}{R}+\pi r \alpha_{b}(r),
$$

where $L=2 l+r \pi$ corresponds to the cavity length and $\mathrm{R}$ is the reflectivity of the mirrors. The simulation (described in Sect. 2) was repeated with the modified equation $(l$ is fixed to $100 \mu \mathrm{m})$, and assuming a facet reflectivity of $R=0.31$. The measurements show a good match between the experimental and simulation results. A minimum value of the threshold current of $34 \mathrm{~mA}$ is obtained for a ring radius of $150 \mu \mathrm{m}$. Surprisingly, some devices show threshold currents and bending losses lower than the simulated values. An explanation for this might come from the additional mode confinement provided by the gain guiding which was not considered in the simulations.

\section{CONCLUSIONS}

We have reported on the design and technological challenges in fabricating small radius semiconductor ring lasers. Simulations indicate that dry etching depth needs to be controlled within a few tens of microns to achieve adequate control over the output power coupling and to minimise the ring dimensions. This key technological issue was addressed by developing a highly selective dry etching process on Aluminium quaternary material systems. An AlInAs core layer acts as a very effective stop etch layer and provides very smooth etched surfaces. The effectiveness of the etching process was assessed on half-ring structures emitting at $1300 \mathrm{~nm}$, yielding current thresholds as low as $34 \mathrm{~mA}$ on $150 \mu \mathrm{m}$ ring radii. The etching process proved also successful on Al-quaternary $1550 \mathrm{~nm}$ material systems. A new wafer design ${ }^{5}$ provided theoretical threshold values of $60 \mathrm{~mA}$ on ring radii as low as $100 \mu \mathrm{m}$ with a minimum threshold of $20 \mathrm{~mA}$ for $170 \mu \mathrm{m}$ radius ring lasers.

\footnotetext{
${ }^{\ddagger} \mathrm{A}$ half ring laser comprises a half ring shaped waveguide (bend radius of $r$ ) joined with two straight sections (length of $l$ ) ending in cleaved facets forming a Fabry-Perot cavity.
} 


\section{ACKNOWLEDGMENTS}

S.F. would like to acknowledge EPSRC (Engineering and Physical Sciences Research Council, UK) for financial support.

\section{REFERENCES}

1. M. Sorel, P. J. R. Laybourn, G. Giuliani and S. Donati, "Unidirectional bistability in semiconductor waveguide ring lasers," Appl. Phys. Lett. 80(17), pp. 3051-3053, 2002.

2. M. Sorel, G. Giuliani, A. Scirè, R. Miglierina, S. Donati, and P. J. R. Laybourn, "Operating regimes of GaAs-AlGaAs semiconductor ring lasers: Experiment and model," IEEE Journal of Quantum Electronics 39(10), pp. 1187-1195, 2003.

3. Hill, Martin T. and Dorren, Harmen J. S. and De Vries, Tjibbe and Leijtens, Xaveer J. M. and Den Besten, Jan Hendrik and Smalbrugge, Barry and Oel, Yok-Siang and Binsma, Hans and Khoe, Giok-Djan and Smit, Meint K., "A fast low-power optical memory based on coupled micro-ring lasers," Nature 432, pp. 206-209, 2004.

4. T. Higashi, S. J. Sweeney, A. F. Phillips, A. R. Adams, E. P. OReilly, T. Uchida, and T. Fujii, "Observation of Reduced Nonradiative Current in $1.3 \mu \mathrm{m}$ AlGaInAs-InP Strained MQW Lasers," IEEE Photon. Tech. Lett. 11(4), pp. 409-411, 1999.

5. H. Y. Wong, M. Sorel, A. C. Bryce, J. H. Marsh, and J. M. Arnold, "Monolithically Integrated InGaAs-AlGaInAs MachZehnder Interferometer Optical Switch Using Quantum-Well Intermixing," IEEE Photon. Tech. Lett. 17(4), pp. 783-785, 2005.

6. P. W. A. Mcllroy, A. Kurobe, and Y. Uematsu, "Analysis and application of theoretical gain curves to the design of multi-quantum-well lasers," IEEE Journal of Quantum Electronics QE-21, pp. 1958-1963, 1985.

7. J. M. Senior, Optical fiber communications: Principles and practice, Prentice Hall, London, $2^{\text {nd }}$ ed., 1992.

8. K. Shinoda, K. Nakahara, and H. Uchiyama, "InGaAlAs/InP ridge-waveguide lasers fabricated by highly selective dry etching in $\mathrm{CH}_{4} / \mathrm{H}_{2} / \mathrm{O}_{2}$ plasma," in International Conference Indium Phosphide and Related Materials, pp. 550-553, (Santa Barbara, CA, United States), May 12-16 2003.

9. M. M. Raj, J. Wiedmann, S. Toyoshima, Y. Saka, K. Ebihara, and S. Arai, "High-Reflectivity Semiconductor/Benzocyclobutene Bragg Reflector Mirrors for GaInAsP/InP Lasers," Journal of Applied Physics 40, pp. 22692277, 2001. 